\title{
Tuning of Continuous-Time Filters Using Genetic Algorithms
}

\author{
Y. Sun ${ }^{*}$ B. T. Chan ${ }^{* *}$ and H. W. Su${ }^{* *}$ \\ Department of Electronic, Communication and Electrical Engineering, Faculty of Engineering and \\ Information Sciences, University of Hertfordshire, Hatfield, Herts, AL10 9AB, U.K. \\ "Tel: ++44 (0)1707 284196, Fax: ++44 (0)1707 284199, Email: Y.Sun@herts.ac.uk \\ "Email: b.t.chan@aherts.ac.uk

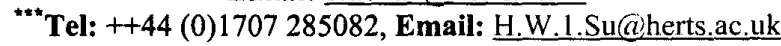

\begin{abstract}
A tuning method of continuous-time filters is proposed which is based on Genetic Algorithms. Simulation results show that the method can be used to tune OTA-C filters for a given accuracy within a reasonable time.
\end{abstract}

\section{Introduction}

Continuous-time (CT) filters [1] have advantages over sampled-data and digital filters in terms of high speed and low power dissipation. Continuous-time OTA-C and MOSFET-C filters have been widely used in video signal processing, wireless communications systems and computer systems. Tuning is crucial for CT filters to overcome the effects of tolerance, device non-ideality, parasitic effects, temperature, environment and ageing [2, 3]. Several tuning methods have been proposed in recent years, including the master-slave, phase-locked loop and adaptive techniques.

Genetic Algorithms (GAs) have proved to be a very powerful search method for many application areas. Genetic Algorithms work on an (binary) encoding of the parameter set, use a population of points in parallel, search globally and randomly, have learning capability, and do not require function derivatives. They thus fit the tuning problem naturally $[4,5]$. This paper introduces a tuning method for CT filters using GAs.

\section{Filter Tuning Method Using GAs}

Optimisation seeks to improve performance towards some optimal point. There are three main types of search methods: calculus-based, enumerative and random. Calculus-based search methods can only find the local maximum and require continuity and derivative existence. In the enumerative search scheme, every time the search algorithm looks at objective function values at every point in the space and thus can only handle the problems with a small number of possibilities. Random search methods such as the simulated annealing and GAs can overcome these shortcomings and have gained wide popularity.
Genetic Algorithms are random global search algorithms. They are based on the idea of natural selection, a biological process in which the fitter individuals will survive in the competing environment. The population formed by individuals will improve from generation to generation until a population is arrived at, containing one or more good solutions. The whole idea is that the fittest of the generations will survive.

When applying GAs to tune filters, we need to define the objective function. Take the second-order low-pass OTA-C filter in Figure 1 as an example. The transfer function of the filter can be found as

$$
\mathrm{H}_{\mathrm{r}}(\mathrm{s})=\mathrm{g}_{\mathrm{m}}{ }^{2} /\left(\mathrm{sC}_{1} \mathrm{C}_{2}+\mathrm{sC}_{1} \mathrm{~g}_{\mathrm{m}}+\mathrm{g}_{\mathrm{m}}{ }^{2}\right)
$$

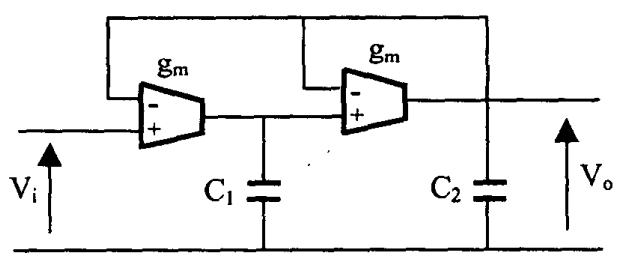

Figure 1. Second-order low-pass OTA-C filter.

The desired second-order low-pass filter is normally written as

$$
H_{d}(s)=K \omega_{0}{ }^{2} /\left(s^{2}+s \omega_{0} / Q+\omega_{0}{ }^{2}\right)
$$

where $\mathrm{K}$ is the $\mathrm{DC}$ gain, $\omega_{0}$ is the cut-off frequency and $\mathrm{Q}$ is the quality factor.

The aim of tuning is to minimise the difference between the desired and real magnitude responses. The objective function can therefore defined as

$$
\varepsilon=\sum_{k=1}^{N}\left(\left|H_{r}\left(j \omega_{k}\right)\right|-\left|H_{d}\left(j \omega_{k}\right)\right|\right)^{2}
$$

where $\varepsilon$ is the sum of squared errors at sampling frequencies $\omega_{k}$ and $N$ is the number of the sampling frequencies. 


\section{Simulation Parameters and Results}

A software tool for the proposed tuning method was written in MATLAB. Many simulations have been conducted for low-pass and band-pass OTA-C filters. For the low-pass filter, for example, the relevant parameters are given below.

The input filter parameters are:

1) Gain $\mathrm{K}=1$.

2) Cut-off frequency fo $=1 \mathrm{MHz}$.

3) Quality factor $Q=1.5$.

4) Range of frequencies to be considered $3 \mathrm{f}_{0}=3 \mathrm{MHz}$.

5) Sampling frequency intervals $\mathrm{f}_{\mathrm{i}}=10 \mathrm{kHz}$.

6) Component ranges $C_{1}, C_{2} \in\left[10^{-12} 10^{-6}\right]$ and $g_{m} \in\left[10^{-5}\right.$ $\left.10^{-2}\right]$.

7) Expected error/accuracy $10^{-6}$.

GA parameters are chosen as:

1) Population size - 100 chromosomes per generation.

2) Number of bits per filter element - 30 bits (so there are approximate $10^{9}$ combinations for each element.).

3) Maximum number of generations - 100.

GA operators used are given below:

1) Coding method - binary coding.

2) Types of coding - Gray coding.

3) Ranking - the ranking algorithm assumes the objective function to be minimised. Giving the fittest chromosome a fitness value of 2 and the least fit chromosome a fitness value of 0 .

4) Selection method - Stochastic Universal Sampling.

5) Crossover method - single-point crossover with crossover rate 0.7 . The chromosomes in the odd numbered positions are crossed with the chromosomes in the adjacent even numbered positions.

6) Mutation rate -0.001 . There are nine thousands of bits per generation. Nine bits are inverted or up to nine new chromosomes are created.

7) Reinsertion method - fitness-based insertion.

Simulation results are presented in Figure 2. For the low-pass filter with the $1 \mathrm{MHz}$ cut-off frequency and the peak producing $Q$ value of 1.5 , the accuracy of $10^{-6}$ in the frequency range of $3 \mathrm{MHz}$ has been achieved within less than 30 generations. The component values are tuned to $\mathrm{C}_{1}=776.81 \mathrm{pF}, \mathrm{C}_{2}=1747.5 \mathrm{pF}$, and $\mathrm{g}_{\mathrm{m}}=7.3 \mathrm{mS}$.

In the example, the frequency range chosen is three times the cut-off frequency as this can cover the whole pass-band as well as the transition-band and stop-band. A uniform sampling interval is used for the whole frequency range. Certainly, variable sampling intervals can be used. Normally a few samples may be needed in the flat passband and stop-band, and more samples in the transitionband. For $Q>0.707$, because of the peak, more sampling points may be needed at the region around the cut-off frequency.

Simulation time is the most important constraint for tuning a filter. There are many factors which influence the computation amount such as the sampling frequency range and intervals, the range of component values $C_{1}, C_{2}$ and $g_{m}$ (the search space) and the accuracy of tuning required. Most of the simulations carried out including the one presented above were finished within a few minutes.
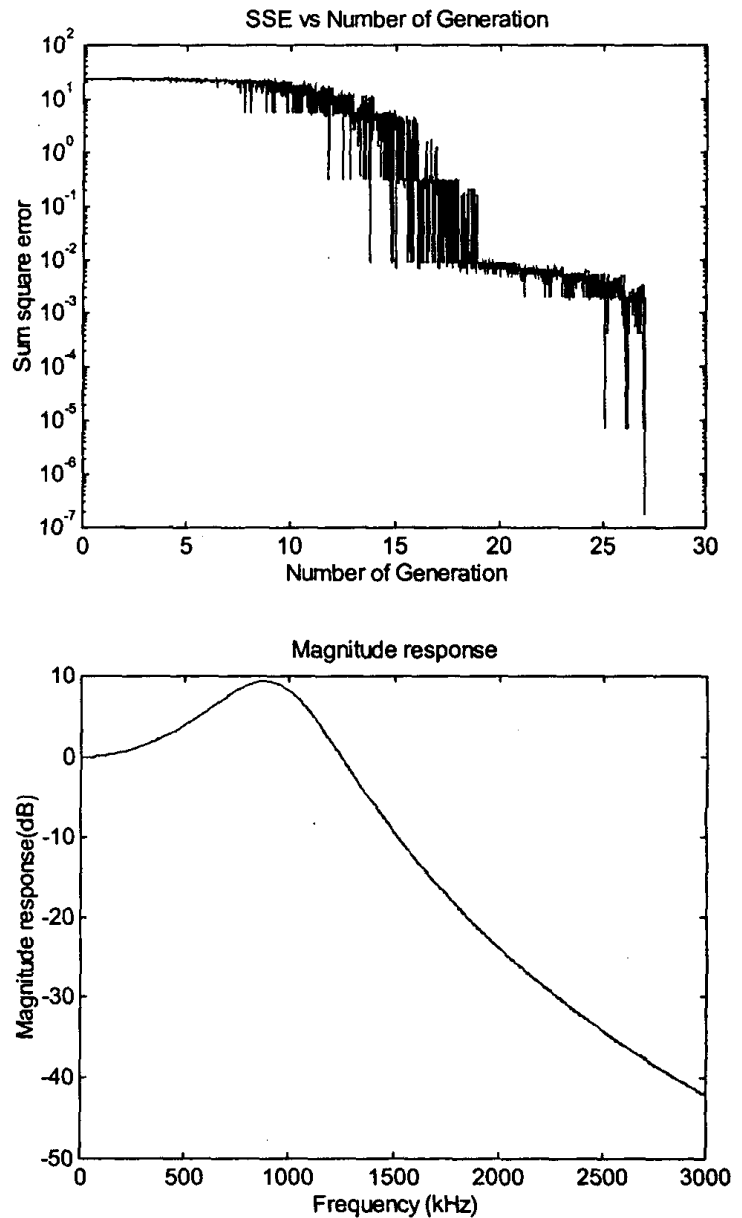

Solid line - real response, dotted line - desired response Figure 2. Simulation results of Sum of Squared Errors (SSE) against number of generations and filter magnitude response for $Q=1.5$ and $f_{0}=1 \mathrm{MHz}$ (Calculated component values are $C_{1}=7.7681 \mathrm{e}-10, C_{2}=1.7475 \mathrm{e}-9, g_{m}=0.0073$ ).

\section{Conclusions}

A tuning method for continuous-time filters using GAs has been proposed. Various low-pass and band-pass OTA- 
C filters have been tuned using the method. Good performances have been achieved. Results suggest that Genetic Algorithms can be applied and may provide a better alternative for continuous-time filter tuning.

\section{References}

[1] T. Deliyannis, Y. Sun and J. K. Fidler, Continuostime active filter design, CRC Press, Florida, USA, ISBN: 0-8493-7893-1, Jan. 1999.

[2] A. I. Karsilayan and R. Schaumann, "A mixed-mode automatic tuning scheme for high- $Q$ continuous-time filters", IEE Proceedings: Circuits, Devices and Systems, vol. 147, no.1, 2000 (Special Issue on High-frequency Integrated Analogue Filters).

[3] K. Loh, D. L. Hiser, W. J. Adams, and R.L. Geiger, "A digitally controlled continuous-time filter structure with wide range and fine resolution capability", IEEE Trans. Circuits Syst., vol. 39, no.5, pp.265-276, 1992.

[4] Y. Sun and W. K. Lau, "Evolutionary tuning method for automatic impedance matching in communication systems", Proc. IEEE Int. Conf. on Electronics, Circuits and Systems, pp.73-77, Portugal, Sept. 1998.

[5] D. H. Horrocks and Y. M. A. Khalifa, "Genetically derived filter circuits using preferred value components", IEE Colloquium on Analogue Signal Processing, Oxford, UK, 1994. 